\title{
Génétique et systématique évolutives du complexe d'espèces Sphaeroma hookeri Leach, Sphaeroma levii Argano et Sphaeroma rugicauda Leach (Crustacés, Isopodes Flabellifères)
}

\section{Génétique formelle de onze locus enzymatiques}

\author{
M. LAULIER \\ Université du Maine, Faculté des Sciences, Laboratoire de Biologie Animale, \\ 72017 Le Mans Cedex, France
}

\begin{abstract}
Résumé
Le déterminisme génétique de six activités enzymatiques (fumarase FU, malate déshydrogénases MDH, glucose phosphate isomérase GPI, phosphatases ALPH, estérases EST, amylases AMY), a été analysé par électrophorèse sur gel de polyacrylamide. L'interprétation repose sur l'analyse de descendances issues de croisements intra- et interspécifiques entre espèces du genre Sphaeroma : S. levii, $S$. rugicauda et sous-espèces : $S$. hookeri hookeri et $S$. $h$. mediterranea. Selon le type d'activité considéré, les résultats démontrent l'existence d'un ou de plusieurs locus : FU, 1 locus monomorphe, GPI : 1 locus polymorphe, MDH : 2 locus monomorphes, ALPH : 2 locus polymorphes, AMY : 2 locus polymorphes liés, EST : 3 locus polymorphes. L'observation d'hétérozygotes permet des inférences quant à la structure quaternaire de l'enzyme active : monomérique pour les amylases et une estérase, dimérique pour la glucose phosphate isomérase, les phosphatases et une estérase.
\end{abstract}

Mots clés : Crustacés, Isopodes, Sphaeroma, génétique formelle, isozymes.

\section{Summary}

Evolutionary genetics and systematics of the complex of species Sphaeroma hookeri Leach, Sphaeroma levii Argano and Sphaeroma rugicauda Leach (Crustacea, Isopoda Flabellifera). 1. Formal genetics of eleven enzymatic loci

Genetic segregations for six enzymatic activities (fumarase FU, malate dehydrogenase MDH, phosphoglucose isomerase GPI, phosphatases ALPH, esterases EST, amylases AMY) were studied by electrophoresis on polyacrylamide gels in intra and interspecific progenies of the species Sphaeroma levii, S. rugicauda and the two sub-species of S. hookeri: S.h. hookeri and S.h. mediterranea. The loci identified were found to be monomorphic (FU, $\mathrm{MDH}_{1}, \mathrm{MDH}_{2}$ ) or polymorphic (GPI, ALPH $, \mathrm{ALPH}_{2}, \mathrm{AMY}_{1}, \mathrm{AMY}_{2}, \mathrm{EST}_{1}, \mathrm{EST}_{2}$ and $\mathrm{EST}_{3}$ ). The loci $\mathrm{AMY}_{1}$ and $\mathrm{AMY}_{2}$ are linked. The occurrence of heterozygotes allowed inferences on quaternary structure of the active enzyme.

Key words : Crustacea, Isopoda, Sphaeroma, formal genetics, isozymes. 


\section{Introduction}

L'existence de peuplements monospécifiques ou hétérospécifiques, la répartition géographique et écologique ainsi que la possibilité d'obtenir des hybrides interspécifiques dans les conditions expérimentales (LEJUEZ, 1966) font du complexe d'espèces Sphaeroma rugicauda $(S . r$.$) et S$. levii $(S . l$.) et des sous-espèces $S$. hookeri hookeri (S.h.h.) et S.h. mediterranea (S.h.m.) un matériel favorable pour les études de génétique écologique, l'analyse de la variation géographique, la compréhension des relations phylogénétiques ainsi que pour l'évaluation du taux d'hybridation naturelle. Afin d'établir les bases génétiques utilisables pour la résolution de ces problèmes, nous avons étudié le déterminisme génétique de 6 activités enzymatiques (fumarase, malate déshydrogénases, phosphatases, glucose phosphate isomérase, estérases et amylases).

L'interprétation génétique repose sur l'analyse des descendances obtenues au laboratoire à partir de géniteurs provenant de peuplements monospécifiques. Cette démarche a été mise en œuvre chez les Crustacés par plusieurs auteurs (LomHolt \& Christensen, 1970 ; Barlow \& Ridgway, 1971 ; Solignac \& Cariou, 1974 ; Bisol et al., 1977 ; Cariou, 1977 ; Sassaman, 1979 ; Bisol et al., 1981 ; Ruvinsky \& LobKov, 1981 ; Legrand-Hamelin et al., 1982 ; Gilard, 1984 ; Borowsky et al., 1985 ; Oxford, 1986).

\section{Matériel et méthodes}

\section{A. Elevage}

Les couples constitués d'individus appartenant à la même espèce ou à des espèces différentes ainsi que leurs descendances sont élevés au laboratoire dans des conditions standards (LEJUEZ, 1966). Les géniteurs proviennent des stations de Luc-sur-Mer et Roscoff (S.l.), Graye-sur-Mer (S.r.), canal de Caen à la mer (S.h.h.) et de Camargue (S.h.m.). La mort des mâles après la fécondation et des femelles après la ponte ne permet pas leur étude enzymatique. Leur phénotype présumé a été établi à partir des principes classiques d'interprétation des zymogrammes, en tenant compte des phénotypes observés dans les populations et les descendances. Quelques descendances de femelles fécondées dans la nature ont été analysées.

\section{B. Electrophorèse}

Les animaux sont broyés dans un broyeur de Potter réfrigéré ou manuellement dans un mortier. Le volume final de solution (1/2 tampon utilisé pour l'électrophorèse $+1 / 2$ solution de sacccharose à $40 \%$ ) varie entre 80 et $100 \mu$ l en fonction de la taille du Sphérome. Les migrations sont réalisées sur gel de polyacrylamide. Le système est décrit par MAURer (1971, système $n^{\circ} 1$, p. 44-45). Quelle que soit la concentration en acrylamide, le rapport acrylamide bis-acrylamide est constant. La distance de migration est de 5 à $6 \mathrm{~cm}$ sauf pour les amylases (migrations de 9 à $10 \mathrm{~cm}$ ). L'intensité 


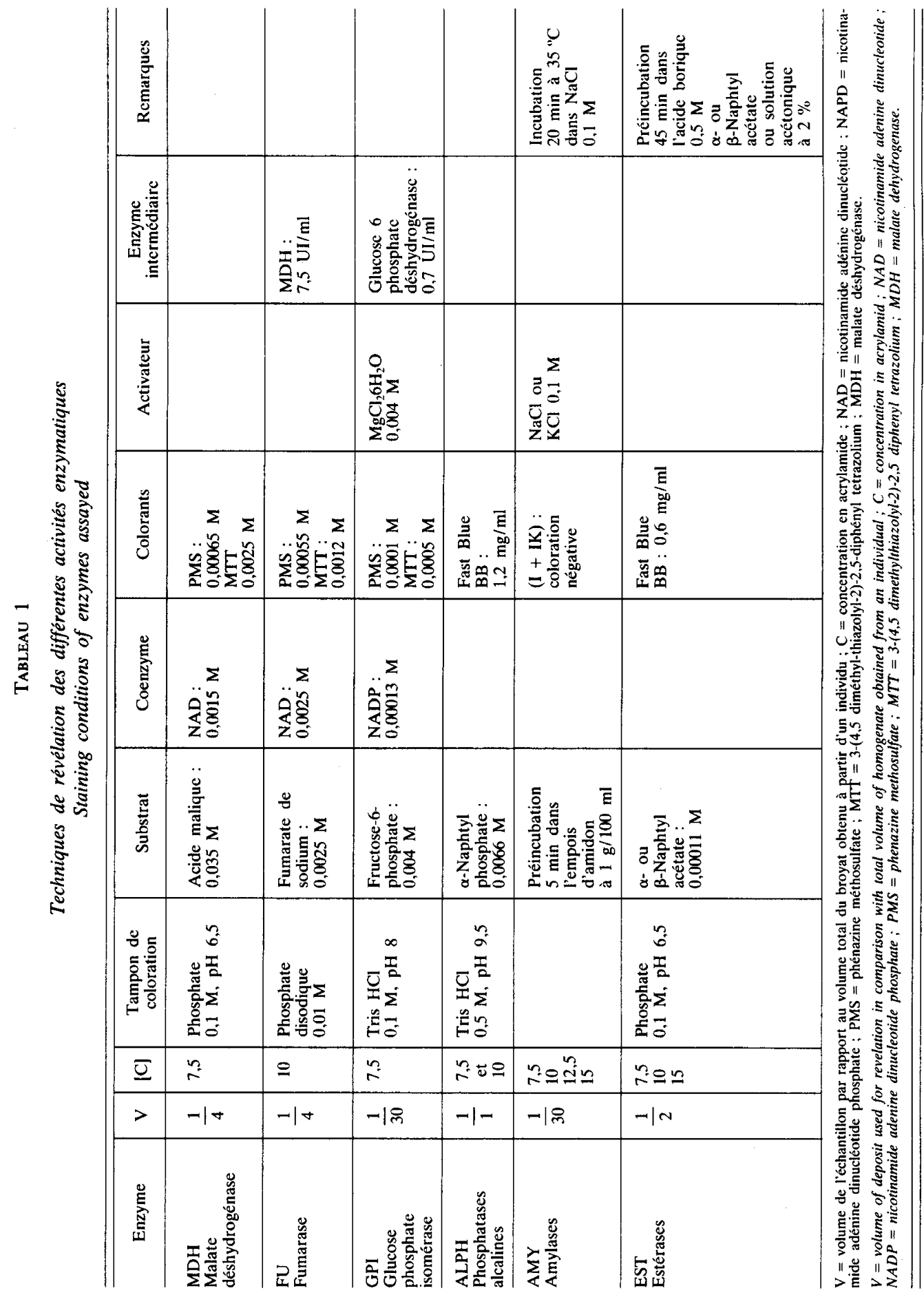


est constante ( $3 \mathrm{~mA}$ par tube). Les techniques de révélation (tableau 1) sont inspirées de Brewer (1970), Maurer (1971), Shaw \& Prasad (1970), Kerambrun (1974) et Cariou (1977). La technique de Hedrick \& SMTth (in Maurer, p. 19-20, 1971) a permis de comparer l'encombrement moléculaire de quelques isozymes.

\section{Nomenclature}

$\mathrm{Si}$ plusieurs isozymes sont révélées, le système le plus éloigné du dépôt porte le $\mathrm{n}^{\mathrm{o}} 1$, les autres, des valeurs croissantes.

La dénomination numérique des allèles en fonction de la mobilité électrophorétique des protéines qu'ils codent a été adoptée. L'allèle de référence (100) correspond à celui observé le plus fréquemment chez les $S$.h. hookeri.

\section{Résultats et interprétation}

\section{A. Fumarase (FU)}

Quel que soit le type de croisement (6 intraspécifiques, 4 interspécifiques), les 60 individus présentent un zymogramme identique : présence d'une seule bande de position constante qui ne permet aucune inférence quant à la structure quaternaire de l'enzyme. Ces observations sont interprétées comme le résultat de l'action d'un seul locus au niveau duquel un seul allèle a été révélé.

\section{B. Malate déshydrogénases $(M D H)$}

Quel que soit le type de croisement (4 intraspécifiques, 4 interspécifiques), les 46 descendants présentent le même zymogramme caractérisé par l'existence de 2 fractions actives invariables. Dans l'hypothèse la plus simple, on admettra l'existence d'au moins 2 locus enzymatiques $M D H_{1}$ et $\mathrm{MDH}_{2}$ comportant chacun un seul allèle.

\section{Phosphatases (ALPH)}

La modification des conditions de révélation des phosphatases démontre que l'ensemble des fractions réagit en milieu acide et alcalin. Les zymogrammes étant plus colorés dans le second cas, nous les qualifierons de phosphatases alcalines.

Quelle que soit l'espèce ou la sous-espèce, le zymogramme présente deux zones d'activité (fig. 1, gels 1 à 5). Le locus $\mathrm{ALPH}_{1}$ se caractérise par une coloration intense et l'absence de polymorphisme intraspécifique ; $\mathrm{ALPH}_{2}$, moins coloré, est polymorphe. 


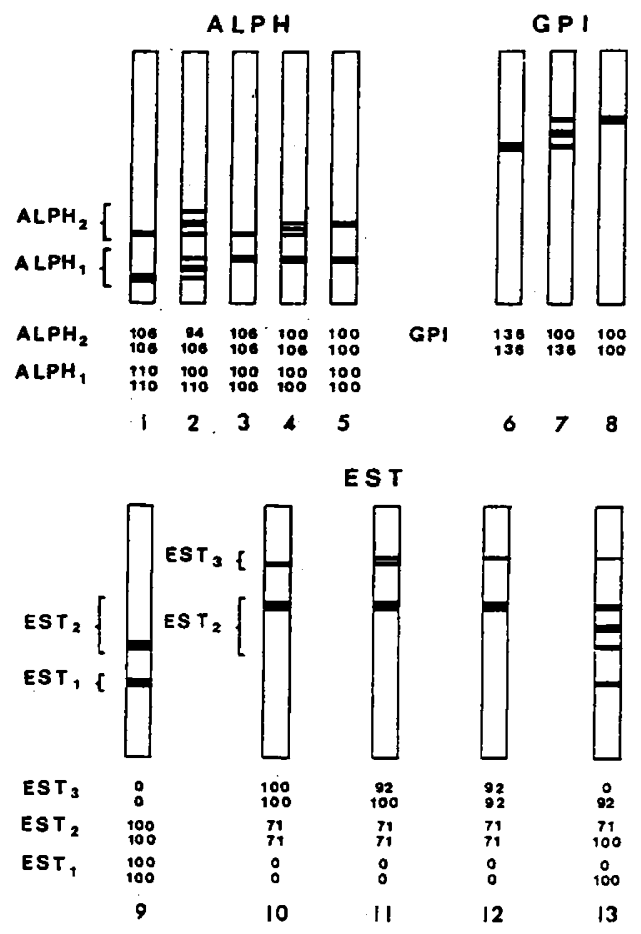

FIG. 1

Représentation schématique de zymogrammes obtenus pour différents systèmes enzymatiques (migrations anodiques). Les génotypes correspondants à chaque phénotype sont indiqués : $A L P H=$ phosphatases alcalines, $G P I=$ glucose phosphate isomérase, EST $=$ estérases.

Diagram of zymograms obtained for different enzymatic systems (anodic migratrions). Examples of phenotypes and genetic interpretation.

$A L P H=$ alkaline phosphatases, $G P I=$ phosphoglucose isomerases, $E S T=$ esterases.

a) Locus $\mathrm{ALPH}$

L'analyse de 8 croisements intraspécifiques (35 jeunes) ou hétérospécifiques hookeri $\times$ rugicauda (7 croisements, 27 descendants), met en évidence l'absence de phénotype nouveau. On observe une seule fraction active (fig. 1, gels 1, 3, 4, 5). Chez les hybrides hookeri $\times$ levii et rugicauda $\times$ levii (8 croisements, 22 descendants), on observe un phénotype à 3 bandes (fig. 1, gel 2). Une des bandes est caractéristique de $S . l$., une autre commune à $S . h$. et $S . r$. et la $3^{\mathrm{e}}$ intermédiaire. Le système $\mathrm{ALPH}_{1}$ est monomorphe à l'intérieur des espèces et comporte deux allèles codominants, l'allèle 110 étant fixé chez $S . l$. et $100 \mathrm{chez} S$.h. et $S$.r. L'existence de trois bandes chez les hybrides hétérozygotes indique une structure dimérique de la protéine active.

\section{b) Locus $\mathrm{ALPH}_{2}$}

On observe 2 catégories d'individus possédant soit 1 bande (fig. 1 , gels $1,3,5$ ) soit 3 (fig. 1, gels 2, 4). L'activité plus forte de la fraction intermédiaire et la localisation 
des bandes extrêmes conduisent à admettre que les individus à 3 bandes sont des hétérozygotes et la molécule active un dimère.

La variabilité des phénotypes observée dans les croisements (tableau 2) s'explique par l'intervention d'un locus dont les 3 allèles 106, 100 et 94 sont codominants.

\section{TABLEAU 2}

$A L P H_{2}$ : distribution des phénotypes dans les descendances et phénotypes parentaux présumés $\mathrm{ALPH}_{2}:$ segregation in progeny and presumed parental phenotypes

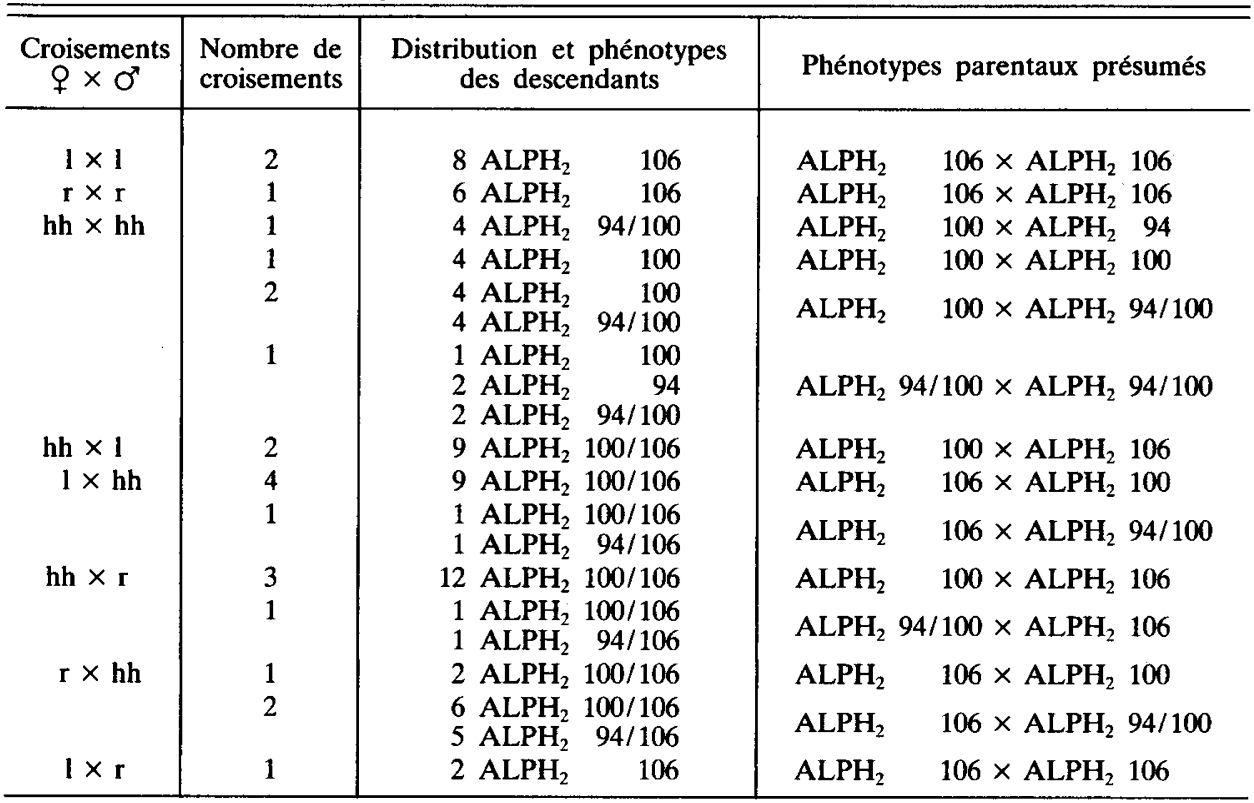

$\mathrm{hh}=S$. hookeri hookeri $; \mathrm{l}=S$. levii $; \mathrm{r}=S$. rugicauda.

\section{Glucose phosphate isomérase (GPI)}

Il existe, comme pour les phosphatases, deux catégories de phénotypes : présence d'une ou de 3 fractions actives (fig. 1, gels 6 à 8). La similitude des observations conduit au même type d'interprétation. Les homozygotes possèdent une bande et les hétérozygotes 3 . La structure de la molécule active est dimérique. Les phénotypes observés dans les descendances (tableau 3) sont compatibles avec l'existence de 4 allèles $157,136,114$ et 100 . 


\section{TABleaU 3}

GPI : distribution des phénotypes dans les descendances et phénotypes parentaux présumés $G P I$ : segregation in progeny and presumed parental phenotypes

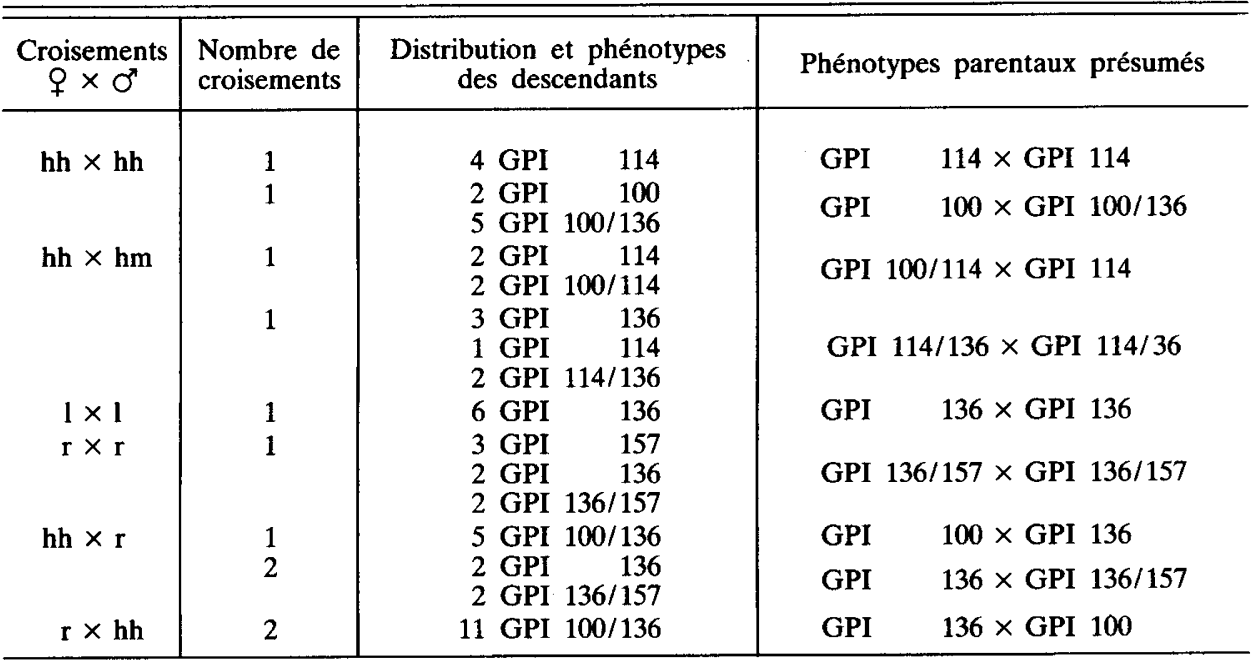

Abréviations identiques au tableau 2 et $\mathrm{hm}=S . h$. mediterranea.

Abbreviations as table 2 and $\mathrm{hm}=\mathrm{S}$.h. mediterranea.

\section{E. Estérases (EST)}

Les résultats des croisements ont permis d'identifier 3 locus (LAULIER, 1981).

\section{a) Locus $E S T_{I}$}

L'allèle 100 est caractéristique de $S . h$. et $S . r$. (fig. 1 , gel 9) et l'allèle nul, 0 , de S.l. (fig. 1, gels $10,11,12$ ). Chez les hétérozygotes $100 / 0$ (fig. 1, gel 13) l'activité est réduite.

\section{b) Locus EST}

L'allèle 100 est commun à S.h. et S.r. (fig. 1, gel 9) et l'allèle 75 caractéristique de S.l. (fig. 1, gels $10,11,12$ ). Les hybrides interspécifiques à 3 bandes (hookeri-levii et rugicauda-levii) indiquent une structure dimérique de l'enzyme active (fig. 1, gel 13).

\section{c) Locus $\mathrm{EST}_{3}$}

Chez S.l., des phénotypes à 2 bandes (fig. 1, gel 11) ou une seule bande (fig. 1, gels 10 et 12 ) ont été observés. Chez $S . h$. et $S . r$, il y a absence d'activité. Il existerait 3 allèles 100,92 et 0 ou allèle nul. 


\section{F. Amylases (AMY)}

Deux zones présentant une forte activité amylasique ont été étudiées. L'existence de variations indépendantes au niveau de chacune de ces zones et les résultats des croisements conduisent à admettre l'existence de 2 locus, $\mathrm{AMY}_{1}$ et $\mathrm{AMY} \mathrm{Y}_{2}$, caractérisés chacun par des phénotypes à 1 ou 2 bandes.

Dans le cas des phénotypes à 2 bandes, chacune migre au niveau de fractions caractéristiques de phénotypes à 1 bande. Ceux-ci correspondent aux homozygotes (fig. 2, gel 1, $A M Y_{1}$ ), ceux à 2 bandes (fig. 2, gels $2,3,4,5,6$ ) aux hétérozygotes. L'enzyme active serait un monomère.

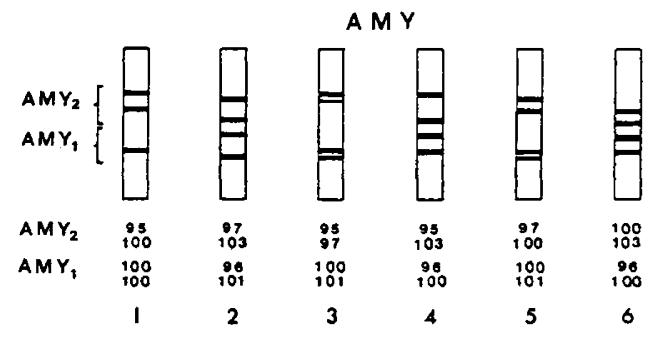

FIG, 2

Représentation schématique de zymogrammes obtenus pour les deux systèmes amylasiques (migrations anodiques). Les génotypes correspondants à chaque phénotype sont indiqués.

Diagram of zymograms obtained for both amylasic systems (anodic migrations).

L'analyse des croisements (tableau 4) confirme ces hypothèses et montre que chaque système est sous la dépendance d'un gène présentant plusieurs allèles codominants. Le locus $\mathrm{AMY}_{1}$ comporte 4 allèles 101, 100, 99 et 96 identifiés dans les croisements et le locus $\mathrm{AMY}_{2}, 5$ allèles 103, 99, 98, 97 et 95.

\section{Liaison $A M Y_{1}, A M Y_{2}$ : encombrement moléculaire}

Dans plusieurs descendances (tableau 4 , croisements ${ }^{*}$ ), on note l'absence de certains phénotypes attendus en cas de ségrégation indépendante. Le nombre limité de descendants n'exclut pas la disparition par simple hasard de certaines catégories mais la liaison des locus $\mathrm{AMY}_{1}$ et $\mathrm{AMY}_{2}$ reste l'interprétation la plus vraisemblable. Le caractère absolu de cette liaison reste à démontrer.

La technique de Hedrick \& SMith a permis de mettre en évidence l'identité d'encombrement moléculaire des différentes isozymes amylasiques. 


\section{TABleAU 4}

$A M Y_{1}$ et $A M Y_{2}$ : distribution des phénotypes dans les descendances et phénotypes parentaux présumés

$A M Y_{1}$ et $A M Y_{2}$ : segregation in progeny and presumed parental phenotypes

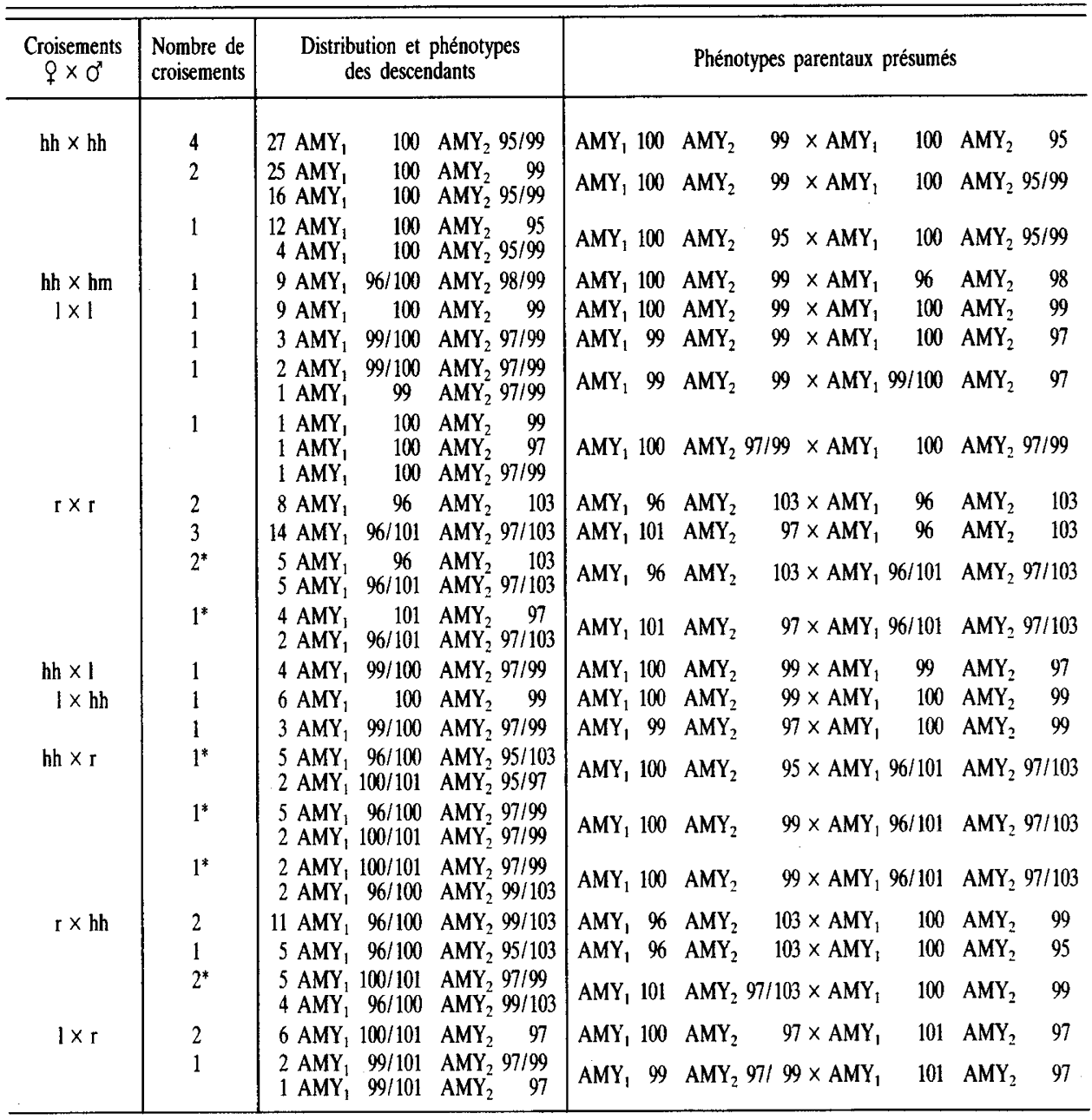

Abréviations identiques aux tableaux 2 et 3 ; ${ }^{*}$ descendances indiquant la liaison des locus $\mathrm{AMY}_{1}$ et $\mathrm{AMY}_{2}$. Abbreviations as tables 2 and 3 ; $^{*}$ progenies indicating the linkage $A M Y_{1}, A M Y_{2}$. 


\section{Discussion}

Les travaux concernant l'analyse enzymatique du genre Sphaeroma sont peu nombreux (Kerambrun, 1970, 1974, 1975 a et b ; CACCONe et al., 1978 ; SBORdoni et al., 1980 ; Edwards \& Heath, 1983 a et b ; Laulier \& Lejuez, 1976, 1977 ; Laulier, $1979,1981,1984)$ et seuls les résultats de Edwards \& HeATH reposent sur une analyse de descendances.

Bien que les comparaisons soient limitées en raison de la variabilité des supports (amidon : Edwards \& Heath, Caccone et al., Sbordoni et al., acrylamide : KeramBRUN, LAULIER, LAULIER \& LeJUEZ), quelques remarques s'imposent.

- MDH : pour CACCONE et al. chez S.h.m., il n'existerait qu'un locus polymorphe ce qui ne concorde pas avec nos observations (2 locus monomorphes).

- ALPH : contrairement à nos résultats et à ceux de Kerambrun, Caccone et al. décrivent chez S.h.m. 2 locus pour les phosphatases acides et 1 pour les alcalines, tous polymorphes. Selon Kerambrun, il existe 2 fractions phosphatasiques majeures présentant un dimorphisme sexuel que nous n'avons pas observé.

- GPI : Caccone et al. observent 1 locus polymorphe chez S.h.m., et chez S.r., Edwards \& Heath décrivent un système diallélique. La présence de 3 fractions chez les hétérozygotes conduit ces auteurs, comme nous-mêmes, à attribuer à cette enzyme une structure dimérique.

- EST : chez S.h.m., Kerambrun décrit 2 fractions majeures, Caccone signale l'existence de 3 locus dont 2 monomorphes. Les 2 fractions observées par Kerambrun et les locus monomorphes analysés par CACCONE pourraient correspondre aux locus $\mathrm{EST}_{1}$ et $\mathrm{EST}_{2}$ que nous décrivons chez $S . h$., le $3^{\mathrm{e}}$ locus de CAccone correspondant à une des nombreuses fractions estérasiques décrites par Kerambrun.

- $\mathrm{AMY}_{1}, \mathrm{AMY}_{2}$ : contrairement aux locus précédents, les amylases n'ont fait l'objet d'aucune étude chez les Sphéromes. Nos résultats mettent en évidence l'identité de l'encombrement moléculaire des différentes isozymes et la liaison des locus. Ces observations peuvent être interprétées comme résultant de la duplication d'un gène ancestral, phénomène observé chez de nombreuses espèces, aussi bien chez les vertébrés (Malacinski \& RutTer, 1969 ; KaRn \& MalacinsKi, 1978) que les invertébrés, Insectes (Paigen, p. 23-28, in Scandalios, 1979), Mollusques (Scheil \& Gunther, 1981) ou Crustacés (OxFord, 1986). Cet auteur décrit chez Asellus aquaticus 6 locus amylasiques dont 5 polymorphes parmi lesquels 3 sont liés. Signalons que nous avons observé 3 zones d'activité amylasique chez les Sphéromes, toutefois la $3^{\mathfrak{e}}$, en raison de sa faible réactivité et de son inconstance n'a pu être analysée génétiquement. Le comportement de cette fraction est peut-être à rapprocher des observations réalisées chez les Crustacés amphipodes du genre Gammarus (Borowsky et al., 1985) chez lesquels des conditions alimentaires et de température différentes permettent "d'induire " l'apparition d'une $3^{\mathrm{e}}$ bande.

Pour plusieurs systèmes enzymatiques, nous avons pu inférer la structure quaternaire de la molécule active : monomérique pour les amylases et une estérase, dimérique pour la glucose phosphate isomérase, les phosphatases et une estérase. On note que les structures quaternaires des protéines étudiées sont identiques à celles rapportées chez les Crustacés par Hedgecock et al. (in Abele, 1982, p. 283-403). Ceci est en accord 
avec l'hypothèse de Ruth \& Wold cités par Hedgecock et al. (in Abele, 1982, p. 283403) selon laquelle « la composition en sous-unités des protéines homologues est de type conservatif chez les organismes supérieurs ».

Si dans de nombreux cas, l'homologie des différentes fractions est facilement établie pour les espèces d'un genre, il n'en est pas de même lorsque de nombreuses isozymes sont révélées. L'analyse des croisements intra- et interspécifiques constitue la méthode d'analyse la plus sûre pour l'établissement des homologies moléculaires, préalable nécessaire à l'évaluation des distances génétiques (LAULIER, à paraître).

Reçu le 15 octobre 1986.

Accepté le 4 mai 1987.

\section{Remerciements}

L'auteur remercie les lecteurs mandatés par la revue pour leurs critiques et suggestions lors de la lecture du manuscrit.

\section{Références bibliographiques}

Abele L.G., 1982. The biology of Crustacea (2), embryology, morphology and genetics. 440 p., Academic Press, New York.

Barlow J., Ridgway G.J., 1971. Polymorphisms of esterase isozymes in the American lobster (Homarus americanus). J. Fisch. Res. Board Can., 28, 15-21.

Bisol P.M., Battaglia B., Bovo G., 1977. Polimorfismi enzymatici in Gammarus insensibilis. 2. Controllo genetico e caratterizzazione delle esterasi. Atti Accad. Naz. Lincei, 62, 261-266.

Bisol P.M., Pasquali P., Varotto V., 1981. Formal genetics of two loci, AP-1 and PGI-1 in the marine copepod Tisbe holothuriae. Vie Milieu, 31, 293-295.

Borowsky R., Borowsky B., Milani H., Greenberg P., 1985. Amylase variation in the salt marsh amphipod, Gammarus palustris. Genetics, 111, 311-323.

Brewer G.J., 1970. Introduction to isozyme techniques. 186 p., Acad. Press, New York.

Caccone H., Cobolli-Sbordoni M., De Matthaeis E., Sbordoni V., 1978. Una datazione su base genetico-molecolare della divergenza tra specie cavernicole e marine di Sferomidi (gen. Monolistra e Sphaeroma, Crustacea, Isopoda). Lav. Soc. Ital. Biogeogr. Nuova Serie, VII, 853-867 (publicato 1982).

CARIOU M.L., 1977. Recherches sur le polymorphisme enzymatique du complexe Jaera albifrons, Leach (Crustacé, Isopode). Thèse de Doctorat d'Etat, Université de Paris VI.

Edwards J.P., Heath D.J., $1983 a$. Dynamics of an enzyme polymorphism in the Isopod, Sphaeroma rugicauda (Leach). 1. Temporal variation in genotype frequencies. Heredity, 51, 467-475.

Edwards J.P., Heath D.J., $1983 \mathrm{~b}$. Dynamics of an enzyme polymorphism in the Isopod, Sphaeroma rugicauda (Leach). 2. Sexual, gametic and fecundity selection. Heredity, 51, 477486.

GILARD J.P., 1984. Contribution à l'étude du polymorphisme enzymatique d'Idotea balthica Pallas (Crustacé Isopode Valvifere). Thèse de $3^{\mathrm{c}}$ cycle, Poitiers.

KaRn R.C., Malacinski G.M., 1978. The comparative biochemistry, physiology and genetics of animal amylases. Adv. Comp. Physiol. Biochem., 7, 1-103. 
Kerambrun P., 1970. Mise en évidence des estérases après électrophorèse sur gel de polyacrylamide chez Idotea balthica, Ligia italica, Sphaeroma serratum, Sphaeroma hookeri et Sphaeroma ghigii (Crustacés Isopodes). C.R. Acad. Sci., 271, 428-441.

Kerambrun P., 1974. Etude de la variabilité biochimique et génétique de Sphaeroma ghigii, $\mathrm{S}$. hookeri et $\mathrm{S}$. serratum (Isopodes Flabellifères). Thèse de Doctorat d'Etat, Faculté des Sciences de Marseille-Luminy.

Kerambrun P., 1975 a. Variabilité biochimique et génétique de Sphaeroma ghigii, S. hookeri et $S$. serratum. Comparaison interspécifique. Biochem. Syst. Ecol., 3, 95-100.

Kerambrun P., 1975 b. Variabilité biochimique et génétique de Sphaeroma ghigii, $S$. hookeri et $S$. serratum. Variations intraspécifiques. Biochem. Syst. Ecol., 3, 101-109.

LAULiER M., 1979. Etude comparative de l'analyse électrophorétique des estérases pour cinq espèces du genre Sphaeroma $: S$. hookeri, $S$. rugicauda, $S$. monodi, $S$. serratum et $S$. teissieri. Cah. Biol. Mar., 20, 417-429.

LAULIER M., 1981. Analyse électrophorétique des systèmes estérasiques chez les hybrides interspécifiques entre trois espèces du genre Sphaeroma, $S$. monodi Bocquet, Hoestlandt, Levi, $S$. rugicauda Leach et $S$. hookeri Leach (Isopodes Flabellifères). Cah. Biol. Mar., 22, 165-175.

LAULIER M., 1984. Essai de génétique et de systématique évolutives de trois espèces du genre Sphaeroma Latreille (Isopodes Flabellifères). Etude du complexe d'espèces interfertiles $\mathrm{S}$. levii Argano, S. hookeri Leach et $\mathrm{S}$. rugicauda Leach. Thèse de Doctorat d'Etat, Université du Mans.

LAulier M., Lejuez R., 1976. Etude comparative de l'analyse électrophorétique de la lactate NAD oxydoréductase pour cinq espèces de Sphéromes Sphaeroma serratum, S. monodi, S. teissieri, S. rugicauda et S. hookeri. C.R. Acad. Sci. Paris, 283, 1779-1781.

Laulier M., Lejuez R., 1977. Analyse électrophorétique de la lactate NAD oxydoréductase chez les hybrides interspécifiques entre trois espèces du genre Sphaeroma : S. monodi, $S$. rugicauda et S. hookeri hookeri. C.R. Acad. Sci. Paris, 284, 2535-2537.

Legrand-Hamelin E., Souty C., Picaud J.L., 1982. Le polymorphisme de la lactate déshydrogénase dans les sous-espèces d'Idotea balthica. Biochem. Syst. Ecol., 10, 79-81.

LEJUEZ R., 1966. Comparaison morphologique, biologique et génétique de quelques espèces du genre Sphaeroma Latreille (Isopodes Flabellifères). Etude de $S$. serratum (Fabricius), de $S$. monodi Bocquet, Hoestlandt, Levi, de $S$. bocqueti, Daguerre de Hureaux, Hoestlandt, Lejuez, de $S$. rugicauda Leach et de $S$. hookeri Leach. Arch. Zool. Exp. Gen., 107, 469-668.

Lomholt B., Christensen B., 1970. Amylase polymorphism in the waterbug, Asellus aquaticus revealed by cellulose acetate electrophoresis. Hereditas, 64, 177-180.

MALACINSKI G.M., RUTTER W.J., 1969. Multiple molecular forms of the $\alpha$-amylase from the rabbit. Biochemistry, 8, 4382-4390.

MAURER H.R., 1971. Disc electrophoresis and related techniques of polyacrylamide gel electrophoresis. 222 p., De Gruyter, Berlin.

OxFord G.S., 1986. Multiple amylase loci in Asellus (Crustacea : Isopoda) : genetics and linkage. Heredity, 56, 105-110.

RUvinSKY A.O., LobKov Y.I., 1981. Esterase genes in Daphnia pulex : linked inheritance and genotypic distribution in natural populations. Theor. Appl. Genet., 60, 113-117.

Sassaman C., 1979. Genetics of the malate dehydrogenase isozymes of the isopod Porcellio scaber. J. Exp. Zool., 210, 507-513.

Sbordoni V., Caccone E., De Matraeis E., Cobolli-Sbordoni M., 1980. Biochemical divergence between cavernicolous and marine Sphaeromidae and the Mediterranean salinity crisis. Experientia, 36, 48-50.

Scandalios J.G., 1979. Physiological genetics. 280 p., Academic Press, New York.

Scheil H.G., Gunther A., 1981. Amylases in Dreissena polymorpha. Pall. (Mollusca : eulamellibranchia) ; evidence for two polymorphic systems. Zool. Ann., 207, 120-122.

Shaw C.R., Prasad R., 1970. Starch gel electrophoresis of enzymes. A compilation of recipes. Biochem. Genet., 4, 297-320.

Solignac M., Cariou M.L., 1974. Données génétiques et structurales sur la lactate déshydrogénase dans la super-espèce Jaera albifons. Biochem. Syst. Ecol., 2, 97-101. 